\title{
Power System Harmonic Estimation Based on Park Transform
}

\author{
Ya Chen*, Tianyao Ji ${ }^{\dagger}$, Mengshi $\mathrm{Li}^{*}$, Qinghua $\mathrm{Wu}^{*}$ and Xuejian Wang*
}

\begin{abstract}
This paper presents a novel method for power system harmonic estimation based on the Park transform. The proposed method firstly extends the signal to a group of three-phase signals in a-b-c coordinate. Then, a linear fitting based method is adopted to estimate the fundamental frequency. Afterwards, the Park transform is utilized to convert the three-phase signals from a-b-c coordinate to d-q0 coordinate. Finally, the amplitude and phase of a harmonic component of interest can be calculated using the $\mathrm{d}$-axis and q-axis components obtained. Simulation studies have been conducted using matrix laboratory (MATLAB) and power system computer aided design/electromagnetic transients including direct current (PSCAD/EMTDC). Simulation studies in MATLAB have considered three scenarios, i.e., no-frequency-deviation scenario, frequency-deviation scenario and the scenario in the presence of inter-harminics. The results have demonstrated that the proposed method achieves very high accuracy in frequency, phase and amplitude estimation under noisy conditions, and suffers little influence of the inter-harmonics. Moreover, comparison studies have proved that the proposed method is superior to FFT and Interpolated FFT with the Hanning Window (IpFFTHW). Finally, a popular case in PSCAD/EMTDC has been employed to further verify the effectiveness of the proposed method.
\end{abstract}

Keywords: Harmonic estimation, Park transform, FFT, Frequency deviation

\section{Introduction}

The increasing use of power electronic devices and nonlinear loads has caused harmonic pollutions and other high frequency contaminations in power systems [1]. Moreover, the harmonics can result in some unexpected effects such as overheating of machines, malfunction of relays and increasing loss in transmission lines [2]. Thus, it is desirable to compensate the harmonic components by injecting the corresponding portion into a power system [3] to relieve the damages that the power system suffers. However, before compensating the corresponding harmonic components, it is essential to estimate the amplitudes and phases of all the harmonics correctly, which is what this paper focuses on.

Frequency domain based methods, such as the discrete Fourier transform (DFT), are commonly used for harmonic analysis. The DFT is simple and can achieve good performance when coping with stable signals. Unfortunately, it tends to suffer some pitfalls, i.e., aliasing, leakage and picket fence phenomena [4], which degrade its accuracy to a great extent. Apart from the frequency domain based methods, some time domain based methods, such as the Kalman filter, have been developed in $[5,6]$ to estimate the amplitudes and phases of harmonics. However, the Kalman filtering approach requires a prior knowledge of the

\footnotetext{
$\dagger$ Corresponding Author: School of Electric Power, South China University of Technology, China. (tyji@scut.edu.cn)

* School of Electric Power, South China University of Technology, China. (\{c.y56, epxjwang\}@mail.scut.edu.cn, \{tyji, mengshili, wuqh\}@scut.edu.cn)

Received: May 17, 2015; Accepted: October 23, 2015
}

statistics of the signal to be processed, and the state matrix needs to be defined accurately as well, leading unpractical in dealing with uncertain signals.

Some evolutional computation based algorithms such as bacterial foraging strategy [7], GA based algorithm [8], GSO based algorithm [4] and PSO based algorithm [3] have also been reported to accomplish the estimation of harmonics in power systems. Despite their feature of stochastic optimization, which makes them unlikely to be trapped in local minima, their mathematical theories are complex and the parameters needed are usually numerous, in comparison with the DFT based methods. Moreover, these types of harmonic estimation algorithms suffer heavy computational burden and time consuming, leading nonexecutable in real time.

Taking all aforementioned problems into consideration, this paper proposes a novel algorithm for harmonic estimation, based on Park transform. This algorithm does not suffer the pitfalls associated with DFT based methods, i.e. aliasing, leakage and picket fence phenomena, and needs less computation burden, compared with Kalman filtering and evolutional computation based methods. Therefore, it can be executable in real time.

The rest of the paper is organized as follows. Section 2 describes the theory of Park transform, section 3 presents Park transform based harmonic estimation algorithm. An attempt for the harmonic estimation under the condition of frequency deviation is also made in this section. Simulation studies have been conducted in section 4. Further, a popular case has been adopted to validate the effectiveness of the proposed method in section 5. Finally, conclusion is drawn at the end of this paper. 


\section{The Theory of Park Transform}

Park transform, as a widely used method of coordinate transform in power systems, was originally proposed by Park $[9,10]$, and usually applicable in three-phase system. The initial motivation of Park transform is to convert the time-variant three-phase flux linkage, armature current and voltage into constant components in the d-q- 0 coordinate, thus simplifying the mathematical model of a synchronous generator [9].

In a balanced three-phase system, the $k^{\text {th }}$ harmonic component of the three-phase voltage or current signal can be expressed as:

$$
\left\{\begin{array}{l}
S_{\mathrm{a}}(t)=A \cos \left(k \omega_{1} t+\varphi_{k}\right) \\
S_{\mathrm{b}}(t)=A \cos \left(k \omega_{1} t+\varphi_{k}-2 \pi / 3\right) \\
S_{\mathrm{c}}(t)=A \cos \left(k \omega_{1} t+\varphi_{k}-4 \pi / 3\right)
\end{array}\right.
$$

where $S_{\mathrm{a}}(t), S_{\mathrm{b}}(t), S_{\mathrm{c}}(t)$ represent the signal in a, b and c axis, respectively. $A$ is the amplitude of the threephase signals, $\omega_{1}=2 \pi f_{1}$, is the fundamental angular frequency, $f_{1}$ is the fundamental frequency, $k$ denotes the harmonic order, $\varphi_{k}$ denotes the initial phase of $S_{\mathrm{a}}(t)$. The three signals can be expressed in an instantaneous space vector as:

$$
S_{\mathrm{abc}}(t)=\left[\begin{array}{c}
S_{\mathrm{a}}(t) \\
S_{\mathrm{b}}(t) \\
S_{\mathrm{c}}(t)
\end{array}\right]
$$

Then the three-phase signal vector in (2) can be transformed from $\mathrm{a}-\mathrm{b}-\mathrm{c}$ coordinate to $\mathrm{d}-\mathrm{q}-\mathrm{0}$ coordinate using the following operator $P_{k^{\prime}}$, which is known as a Park matrix:

$$
P_{k^{\prime}}=\frac{2}{3}\left[\begin{array}{ccc}
\cos \alpha & \cos (\alpha-2 \pi / 3) & \cos (\alpha+2 \pi / 3) \\
\sin \alpha & \sin (\alpha-2 \pi / 3) & \sin (\alpha+2 \pi / 3) \\
1 / 2 & 1 / 2 & 1 / 2
\end{array}\right]
$$

where $\alpha$ is a time varying variable and set as:

$$
\alpha=k^{\prime} \omega_{1} t
$$

with $k^{\prime}$ being an integer. Next, the Park transform of $S_{\mathrm{abc}}(t)$ can be expressed as:

$$
S_{\mathrm{dq} 0}(t)=P_{k^{\prime}} S_{\mathrm{abc}}
$$

where, $S_{\mathrm{dq} 0}(t)$ is a vector in the d-q-0 coordinate and its d, $\mathrm{q}$ and 0 -axis components can be calculated from (5) as:

$$
S_{\mathrm{dq} 0}(t)=\left[\begin{array}{c}
S_{\mathrm{d}}(t) \\
S_{\mathrm{q}}(t) \\
S_{0}(t)
\end{array}\right]=\left[\begin{array}{c}
A_{k} \cos \left[-\varphi_{k}+\left(k^{\prime}-k\right) \omega_{1} t\right] \\
A_{k} \sin \left[-\varphi_{k}+\left(k^{\prime}-k\right) \omega_{1} t\right] \\
0
\end{array}\right]
$$

Noting that if $k^{\prime}$ is set to be the same as $k$, i.e., $k^{\prime}=k$, then (6) can be rewritten as:

$$
S_{\mathrm{dq} 0}(t)=\left[\begin{array}{c}
A_{k} \cos \left(-\varphi_{k}\right) \\
A_{k} \sin \left(-\varphi_{k}\right) \\
0
\end{array}\right]
$$

From (7), it can be seen that the components in $d$ axis and $\mathrm{q}$ axis are constants under the condition of $k^{\prime}=k$. Thus it is easy to conclude that with special condition satisfied, i.e., $k^{\prime}=k$, the three-phase time varying components in a-b-c coordinate can be converted to constant components in $\mathrm{d}-\mathrm{q}-0$ coordinate, which is an attractive feature when simplifying the mathematical model of synchronous machine [9].

In practice, the three-phase signals in power systems usually contain various harmonic components and can be expressed as:

$$
\left\{\begin{array}{l}
S_{\mathrm{a}}(t)=\sum_{k=1}^{m} A_{k} \cos \left(k \omega_{1} t+\varphi_{k}\right) \\
S_{\mathrm{b}}(t)=\sum_{k=1}^{m} A_{k} \cos \left(k \omega_{1} t+\varphi_{k}-2 \pi / 3\right) \\
S_{\mathrm{c}}(t)=\sum_{k=1}^{m} A_{k} \cos \left(k \omega_{1} t+\varphi_{k}-4 \pi / 3\right)
\end{array}\right.
$$

where $m$ represents the highest order of the harmonic component contained in the three-phase signals, $A_{k}$ represents the amplitude of the $k^{\text {th }}$ harmonic component. Then Park transform is utilized to accomplish the coordinate transform and the transformed results are shown as:

$$
\left[\begin{array}{l}
S_{\mathrm{d}}(t) \\
S_{\mathrm{q}}(t) \\
S_{0}(t)
\end{array}\right]=\left[\begin{array}{c}
\sum_{k=1}^{m} A_{k} \cos \left[-\varphi_{k}+\left(k^{\prime}-k\right) \omega_{1} t\right] \\
\sum_{k=1}^{m} A_{k} \sin \left[-\varphi_{k}+\left(k^{\prime}-k\right) \omega_{1} t\right] \\
0
\end{array}\right]
$$

From (9), it can be easily seen that the $k^{\prime}$ th harmonic component in a-b-c coordinate has been transformed to a constant component in $\mathrm{d}-\mathrm{q}-0$ coordinate, whereas the other components in a-b-c coordinate have been converted to harmonic components in $\mathrm{d}-\mathrm{q}-0$ coordinate. This feature can be employed to estimate the harmonic parameters, which will be explained in detail in the next section.

\section{Harmonic Estimation Based on Park Transform}

\subsection{The extension of a single-phase signal to three- phase signals}

As can be seen in section 2, Park transform is only 
applicable to process three-phase signals in a-b-c coordinate. However, the most common harmonic estimation situation is a single-phase scenario, where Park transform seems incapable. To tackle this problem, we can construct a group of three-phase signals from the single-phase signal [11] using the method described below. Firstly, the original single-phase signal is viewed as phase-a signal in $a-b-c$ coordinate. Then phase- $b$ and phase-c signals, can be obtained by lagging the phase of the phase-a signal for $2 \pi / 3$ and $4 \pi / 3$, respectively.

Assuming a single-phase signal $S(t)$ with $m$ harmonic components is expressed as:

$$
S(t)=\sum_{k=1}^{m} A_{k} \cos \left(k \omega_{1} t+\varphi_{k}\right)
$$

As mentioned above, if the $n^{\text {th }}$ harmonic is of interest to be estimated, a group of three-phase signals can be constructed as:

$$
\left\{\begin{array}{l}
S_{\mathrm{a}}(t)=S(t) \\
S_{\mathrm{b}}(t)=\sum_{k=1}^{m} A_{k} \cos \left[k\left(\omega_{1} t-2 \pi / 3 n\right)+\varphi_{k}\right] \\
S_{\mathrm{c}}(t)=\sum_{k=1}^{m} A_{k} \cos \left[k\left(\omega_{1} t-4 \pi / 3 n\right)+\varphi_{k}\right]
\end{array}\right.
$$

Consequently, the three signals obtained construct a group of three-phase signals which can be denoted as a vector:

$$
S_{\mathrm{abc}}(t)=\left[\begin{array}{c}
S_{\mathrm{a}}(t) \\
S_{\mathrm{b}}(t) \\
S_{\mathrm{c}}(t)
\end{array}\right]
$$

\subsection{The proposed harmonic estimation method}

It should be mentioned that the proposed harmonic estimation method consists of two stages: frequency estimation which will be discussed in the next subsection and harmonic estimation, which will be given in this subsection.

After the extension of a single-phase signal to a group of three-phase signals as shown in subsection 3.1, the Park transform can be used to estimate a certain harmonic component contained in the original single-phase signal.

If the $n^{\text {th }}$ harmonic component in the original signal is of interest, we should firstly set $k^{\prime}$ in (4) to be $n$, thus constructing a Park matrix as:

$$
P_{\mathrm{n}}=\frac{2}{3}\left[\begin{array}{ccc}
\cos \alpha_{n} & \cos \left(\alpha_{n}-2 \pi / 3\right) & \cos \left(\alpha_{n}+2 \pi / 3\right) \\
\sin \alpha_{n} & \sin \left(\alpha_{n}-2 \pi / 3\right) & \sin \left(\alpha_{n}+2 \pi / 3\right) \\
1 / 2 & 1 / 2 & 1 / 2
\end{array}\right]
$$

where, $\alpha_{n}=n \omega_{1}$. Then we can divide the constructed three-phase signals in (11) into two parts: the $n^{\text {th }}$ harmonic component denoted as $S_{\mathrm{n}}(t)$ and the summation of all the other harmonic components denoted as $S_{\text {oth }}(t)$ :

$$
\begin{gathered}
S_{\mathrm{abc}}(t)=\mathrm{S}_{\mathrm{n}}(t)+S_{\mathrm{oth}}(t) \\
S_{\mathrm{n}}(t)=\left[\begin{array}{c}
A_{n} \cos \left(n \omega_{1} t+\varphi_{k}\right) \\
A_{n} \cos \left(n \omega_{1} t+\varphi_{k}-2 \pi / 3\right) \\
A_{n} \cos \left(n \omega_{1} t+\varphi_{k}-4 \pi / 3\right)
\end{array}\right] \\
S_{\text {oth }}(t)=\left[\begin{array}{c}
\sum_{k=1, k \neq n}^{m} A_{k} \cos \left(k \omega_{1} t+\varphi_{k}\right) \\
\sum_{k=1, k \neq n}^{m} A_{k} \cos \left[k\left(\omega_{1} t-2 \pi / 3 n\right)+\varphi_{k}\right] \\
\sum_{k=1, k \neq n}^{m} A_{k} \cos \left[k\left(\omega_{1} t-4 \pi / 3 n\right)+\varphi_{k}\right]
\end{array}\right]
\end{gathered}
$$

Let $S_{\mathrm{dn}}(t), S_{\mathrm{qn}}(t), S_{0 \mathrm{n}}(t)$ represent the transformed d-axis, q-axis and 0-axis components of $S_{\mathrm{n}}(t)$, respectively. Similarly, let $S_{\text {doth }}(t), S_{\text {qoth }}(t), S_{0 \text { oth }}(t)$ represent those of $S_{\text {oth }}(t)$, respectively.

Park transform is obviously a linear transformation, thus satisfying the inherent property of a linear transformation:

$$
P S_{12}=P S_{1}+P S_{2}
$$

where $P$ denotes the Park matrix, $S_{12}, S_{1}$ and $S_{2}$ are all $3 \times 1$ column vectors which satisfy $S_{12}=S_{1}+S_{2}$.

According to the linear property of Park Transform, the Park transform of $S_{\mathrm{abc}}(t)$ equals the summation of the Park transform of $S_{\mathrm{n}}(t)$ and $S_{\text {oth }}(t)$ :

$$
\left[\begin{array}{l}
S_{\mathrm{d}}(t) \\
S_{\mathrm{q}}(t) \\
S_{0}(t)
\end{array}\right]=\left[\begin{array}{c}
S_{\mathrm{dn}}(t) \\
S_{\mathrm{qn}}(t) \\
S_{0 \mathrm{n}}(t)
\end{array}\right]+\left[\begin{array}{c}
S_{\mathrm{doth}}(t) \\
S_{\mathrm{qoth}}(t) \\
S_{0 \mathrm{oth}}(t)
\end{array}\right]
$$

where, $S_{\mathrm{d}}(t), S_{\mathrm{q}}(t)$ and $S_{0}(t)$ represent the transformed d-axis, q-axis and 0 -axis components of $S_{\mathrm{abc}}(t)$, respectively. It can be easily derived from (6) and (7) that the first part in the right side of the equality sign in (18) satisfies:

$$
\left[\begin{array}{c}
S_{\mathrm{dn}}(\mathrm{t}) \\
S_{\mathrm{qn}}(\mathrm{t}) \\
S_{0 \mathrm{n}}(\mathrm{t})
\end{array}\right]=\left[\begin{array}{c}
A_{n} \cos \left(-\varphi_{n}\right) \\
A_{n} \sin \left(-\varphi_{n}\right) \\
0
\end{array}\right]
$$

thus, the amplitude and phase of the $n^{\text {th }}$ harmonic component can be calculated as:

$$
\left\{\begin{array}{c}
A_{n}=\sqrt{S_{\mathrm{dn}}^{2}(t)+S_{\mathrm{qn}}^{2}(t)} \\
\varphi_{n}=-\tan ^{-1}\left(S_{\mathrm{qn}}(t) / S_{\mathrm{dn}}(t)\right)
\end{array}\right.
$$


once $S_{\mathrm{dn}}(t)$ and $S_{\mathrm{qn}}(t)$ have been calculated.

Some further processing steps on $S_{\mathrm{d}}(t)$ and $S_{\mathrm{q}}(t)$ can be taken to figure out $S_{\mathrm{dn}}(t)$ and $S_{\mathrm{qn}}(t)$. The processing steps are averaging one-fundamental-cycle samples of $S_{\mathrm{d}}(t)$ and $S_{\mathrm{q}}(t)$, respectively:

$$
\left\{\begin{array}{l}
S_{\mathrm{dn}}^{\prime}=\frac{1}{N} \sum_{k=1}^{N} S_{\mathrm{d}}(k) \\
S_{\mathrm{qn}}^{\prime}=\frac{1}{N} \sum_{k=1}^{N} S_{\mathrm{q}}(k)
\end{array}\right.
$$

where $N$ denotes the number of samples in one fundamental cycle, $S_{\mathrm{dn}}^{\prime}$ and $S_{\mathrm{qn}}^{\prime}$ represent the processed results of $S_{\mathrm{d}}(t)$ and $S_{\mathrm{q}}(t)$, respectively.

Through the processing above, $S_{\mathrm{dn}}^{\prime}$ and $S_{\mathrm{qn}}^{\prime}$ are exactly $S_{\mathrm{dn}}(t)$ and $S_{\mathrm{qn}}(t)$, respectively, which can be proven theoretically. And the detailed proving process is given in Appendix A.

In practice, the proposed harmonic estimation method is usually carried out as follows. Firstly, utilize the fast Fourier transform (FFT) to analysis the original signal and determine the probable range of orders of the harmonic components contained in the original signal. Denote the highest harmonic order as $m$. Secondly, set $n$ in (11), (13) to be $1,2, \ldots, m$ to extend the original signal to three-phase signals and construct different Park matrixes to accomplish the Park transform to estimate the amplitudes of all the harmonic components. However, due to the effect of noise and computation error, even a certain harmonic component ranged from the $1^{\text {st }}$ to the $m^{\text {th }}$ is not contained in the original signal, its amplitude estimated by the proposed method is not zero in fact. Hence, we set a threshold $\varepsilon$ to determine whether the estimated harmonic component is an effective one (i.e., really contained in the original signal) or not. If the estimated amplitude exceeds $\varepsilon$, the corresponding harmonic component is considered as an effective one and calculate its phase, otherwise an ineffective one and there is no need to calculate its phase. Fig. 1 depicts the procedures of the proposed harmonic estimation method.

\subsection{Frequency estimation}

Frequency, as one of the parameters in a power system, plays an important role for many application [12] since it is generally used to indicate the system operation state. Moreover, the frequency can be used as a base for estimating the other parameters, including the amplitude and phase of voltage and current signals [12]. Some literature $[12,13]$ has reported that the frequency deviation can cause great errors in the harmonic estimation using fast Fourier transform (FFT) for the reason that the DFT/FFT based methods cannot detect the frequency deviation, which leads to asynchronous sampling and incorrect estimation results. Superior to the DFT/FFT method, the

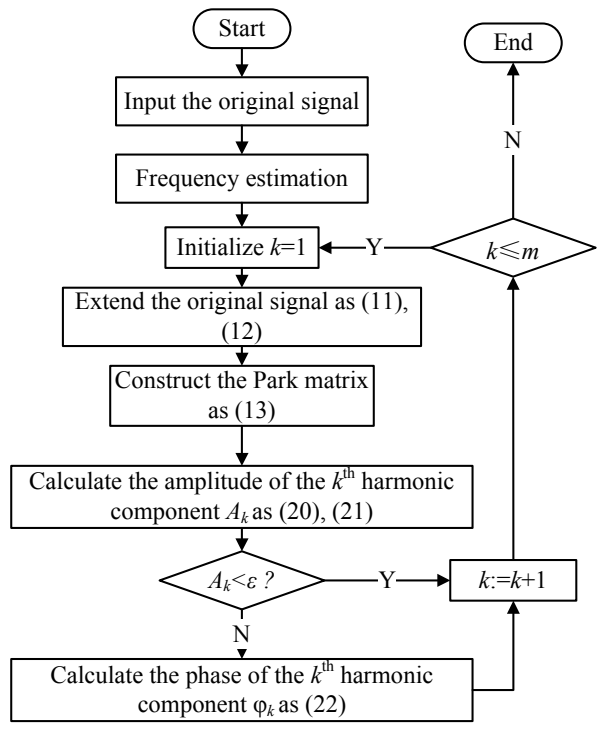

Fig. 1. The flowchart of the proposed method

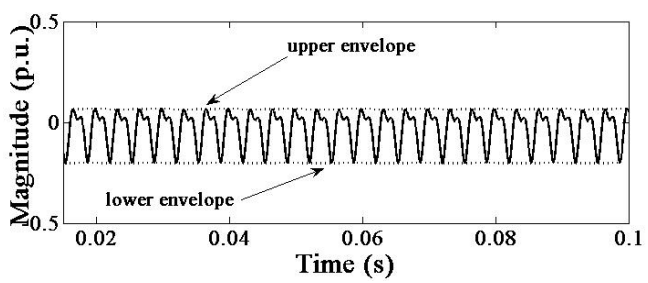

(a)

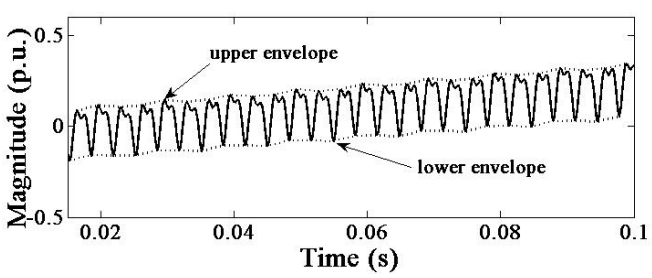

(b)

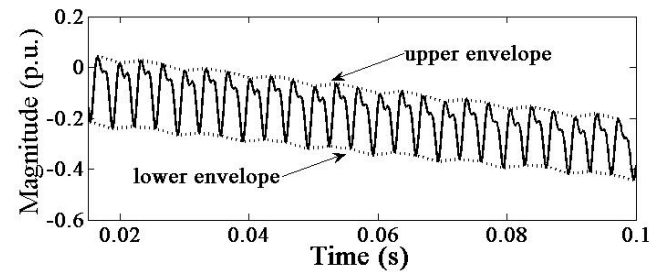

(c)

Fig. 2. The d-axis component of the fundamental frequency component transformed by Park transform: (a) 50 Hz; (b) $50.5 \mathrm{~Hz}$; (c) $49.5 \mathrm{~Hz}$.

proposed method can detect the fundamental frequency deviation and estimate the fundamental frequency before estimating the amplitudes and phases of the harmonic components. Here, we introduce a linear fitting method to detect and estimate the deviated frequency.

By trial and error, we find that if no frequency deviation happens, the upper or the lower envelope (or the tendency) of the transformed d-axis component of the fundamental 
frequency component stays flat, but once the frequency deviation happens, the envelope will become increasing or decreasing. Assume the rated fundamental frequency is 50 Hz. Fig. 3 shows the d-axis component of the fundamental frequency component with the fundamental frequencies of $50 \mathrm{~Hz}, 50.5 \mathrm{~Hz}$ and $49.5 \mathrm{~Hz}$, respectively.

To quantitatively describe the features above, a least square (LS) method is adopted to fit the samples in onefundamental cycle of the d-axis component and the fitting function is a linear function $y=a x+b$, where $x$ is the time variable, $a$ and $b$ are the first-order coefficient and constant term, respectively. $a$ can reflect whether the upper and lower envelop mentioned above is flat or not, and it can be proven theoretically that under the condition of no frequency deviation, $a$ is very close to zero, which can be seen in Appendix B. If $a$ does not equal zero, it means that frequency deviation happens. Assume the samples in one fundamental cycle are $s(1), s(2), \ldots, s(N)$. The aim of fitting the $N$ samples using LS with the function $y=a t+b$ is to figure out the optimal $a$ and $b$ to minimize the quadratic sum of the error:

$$
\sum_{k=1}^{N}(y(k)-s(k))^{2}
$$

As mentioned above, when no frequency deviation occurs in power systems, the upper or the lower envelope of the d-axis component of the fundamental frequency component stays flat. Consequently, the first-order coefficient $a$, i.e., the slope of the line $y=a x+b$, is close to 0 in this case. When frequency deviation occurs, the upper envelope or the lower envelope presents an increasing or a decreasing tendency, which will no doubt lead the absolute value of the slope $a$ considerably larger than that under the condition of no frequency deviation. Thus, we can set a proper threshold $\gamma$ to determine whether the fundamental frequency we estimate differs greatly from the real fundamental frequency. If the absolute value of the slope $a$ exceeds $\gamma$, it means that the a fundamental frequency deviation has occurred, otherwise, no frequency deviation has occurred. If a frequency deviation has occurred, we can adjust the frequency to make it change towards the real deviated system frequency. Denote the frequency we are adjusting as $f_{e}$, the real system frequency as $f_{1}$, and the frequency estimation resolution as $\Delta f$ which is set adaptively according to $|a|$ at the current frequency adjusting step. Then the frequency estimation is carried out as follows.

1) Initialize $f_{e}$ and $\Delta f$ as $50 \mathrm{~Hz}$ and $0.1 \mathrm{~Hz}$, respectively.

2) Utilize Park transform to obtain the d-axis component of the fundamental frequency component and calculate $|a|$ and $\Delta f$. Then compare $|a|$ with the predefined threshold $\gamma$ to determine whether a frequency deviation has occurred.

3) If $|a|>\gamma$, the first adjusting step is $f_{e}=f_{e}-\Delta f$ and

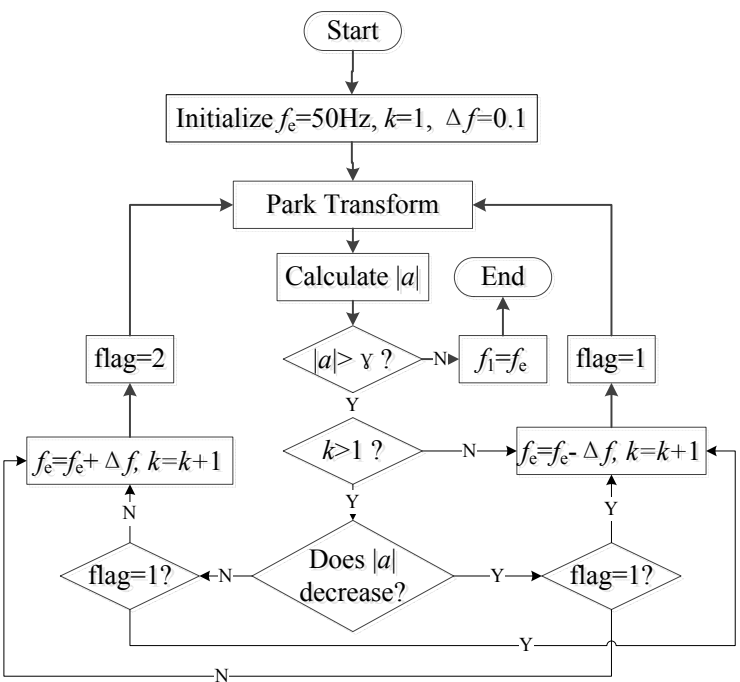

Fig. 3. The flowchart of the frequency estimation

then go back to step 2). If the current adjusting step is not the first adjusting step, compare the current value of $|a|$ with that of the latest previous step. If the value of $|a|$ increases, change the adjusting direction of the $f_{e}$, i.e., take $f_{e}=f_{e}-\Delta f$ as the current adjusting step if the latest previous step is $f_{e}=f_{e}+\Delta f$ or take $f_{e}=f_{e}+\Delta f$ as the current adjusting step if the latest previous step is $f_{e}=f_{e}-\Delta f$; otherwise, keep the same adjusting direction. Then go back to step 2).

4) If $|a| \leq \gamma$, it means the frequency has been adjusted close to the real deviated frequency and the current adjusted frequency is considered to be the optimal estimation of the deviated frequency.

Finally, the adjusting procedures are shown as following flowchart Fig. 3. In Fig. 3, $k$ is an integer variable to indicate the order of the current frequency adjusting step. The variable flag is to record the direction of the latest previous adjusting step ( 1 for $f_{e}=f_{e}-\Delta f$ and 2 for $\left.f_{e}=f_{e}+\Delta f\right)$.

\section{Simulation Studies}

\subsection{Simulation model}

In order to conduct simulation studies, a simulation model which has been frequently employed [14-16] is adopted in this paper to generate a test signal here. Fig. 4 shows the structure of the simulation model.

The model above is built in MATLAB. As can be seen from Fig. 4, it is a two-bus three-phase system with a fullwave six pulse bridge rectifier at the load bus. The system fundamental frequency is $50 \mathrm{~Hz}$. The test signal for simulation is a distorted voltage waveform sampled at the terminals of the load bus. The contents of harmonics in the 
Table 1. The contents of harmonics in the test signal

\begin{tabular}{c|c|c}
\hline Harmonic order & Amplitude (p.u.) & Phase (Degree) \\
\hline 1 & 0.95 & -2.02 \\
\hline 5 & 0.09 & 82.1 \\
\hline 7 & 0.043 & 7.9 \\
\hline 11 & 0.03 & -147.1 \\
\hline 13 & 0.033 & 162.6 \\
\hline \multicolumn{3}{|c}{ Bus 1 2 6 Pulse Rectifier } \\
Generator & Transfer Impedance & Load \\
& &
\end{tabular}

Fig. 4. The simulation model

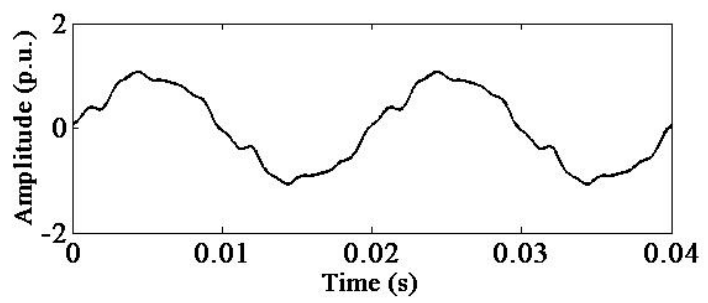

Fig. 5. The waveform of the test signal

test signal is shown in Table 1 and the waveform of the test signal in two fundamental cycles is plotted in Fig. 5.

\subsection{Parameters setting}

In this section, several parameters are set. The sampling rate $f_{s}$ is set to be $25 \mathrm{kHz}$, i.e., 500 samples per fundamental cycle. To make the simulation more practical two different levels of white Gaussian noises (WGNs) have been added to the original signal and the signal-noise-ratio (SNR) of the additive noises are set to $40 \mathrm{~dB}$ and $30 \mathrm{~dB}$, respectively.

The threshold $\varepsilon$ in section 3.2 is set to be 0.005 in consideration of the effect of noise and the calculating error. The threshold $\gamma$ in section 3.3 is set to 0.2 which cannot only guarantee the accuracy of the frequency estimation but also sensitivity of the frequency deviation detection.

The frequency estimation resolution $\Delta f$ is set adaptively. When the slope $|a|$ is very large, which indicates a relatively great deviation from the real fundamental frequency, $\Delta f$ should be set reasonably large in order to accelerate the moving speed of the estimated frequency towards the real fundamental frequency. On the other hand, when the slope $|a|$ becomes very little, which means a small deviation from the real fundamental frequency, $\Delta f$ should be set reasonably little, in order to make the estimated frequency slowly get close to the real fundamental frequency, thus avoiding making the adjusting frequency move over the real fundamental frequency in the right direction. In order

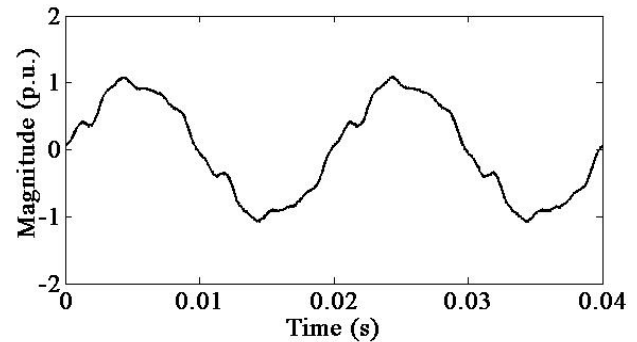

(a)

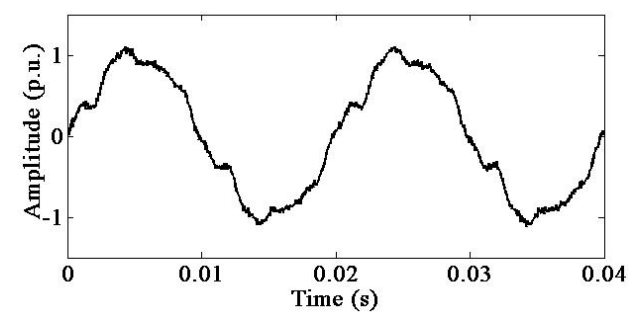

(b)

Fig. 6. The waveforms of the noisy signals: (a) $40 \mathrm{~dB}$; (b) $30 \mathrm{~dB}$.

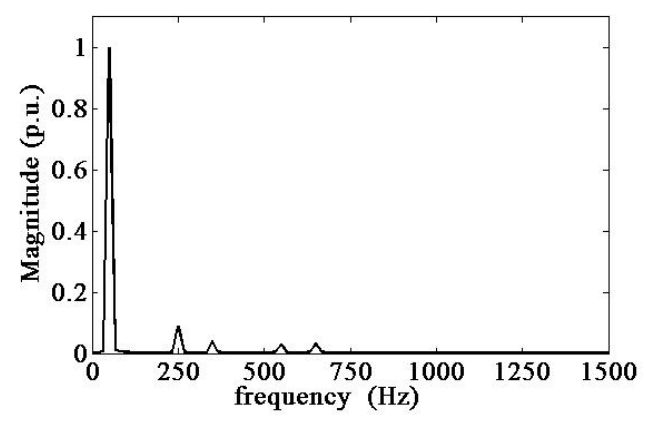

Fig. 7. The FFT analysis of the test signal

to satisfy the two conditions above, $\Delta f$ is formulated as:

$$
\Delta f=0.2 e^{-\frac{1}{5|a|}}
$$

\subsection{Simulation results under the condition of no frequency deviation}

The noisy test signals are plotted in Fig. 6. A FFT analysis is conducted beforehand to determine the probable range of the harmonic orders and the result is shown as Fig. 7. It can be seen from Fig. 7 that the highest harmonic component is under $750 \mathrm{~Hz}$. Thus, we just need to estimate the harmonic components whose frequencies are within $750 \mathrm{~Hz}$.

The estimated amplitudes are shown in Table 2, and the amplitudes of the effective harmonic components, whose amplitudes exceed the predefined threshold $\varepsilon$, i.e., 0.005 , are highlighted in bold. Compare Table 1 with Table 2, it can be found that the proposed method has detected all the harmonic components contained in the test signal and 
Table 2. The estimated amplitudes of the test signal with different levels of noise

\begin{tabular}{c|c|c|c}
\hline $\begin{array}{c}\text { Harmonic } \\
\text { order }\end{array}$ & \multicolumn{3}{|c}{ Amplitude (p.u.) } \\
\hline & no noise & $40 \mathrm{~dB}$ & $30 \mathrm{~dB}$ \\
\hline 1 & $\mathbf{0 . 9 5 0 0}$ & $\mathbf{0 . 9 4 9 9}$ & $\mathbf{0 . 9 4 9 5}$ \\
\hline 2 & $1.17 \times 10^{-16}$ & $6.23 \times 10^{-4}$ & 0.0018 \\
\hline 3 & $6.05 \times 10^{-17}$ & $8.43 \times 10^{-4}$ & $9.28 \times 10^{-4}$ \\
\hline 4 & $2.75 \times 10^{-16}$ & $4.73 \times 10^{-4}$ & 0.0014 \\
\hline 5 & $\mathbf{0 . 0 8 9 9}$ & $\mathbf{0 . 0 8 9 8}$ & $\mathbf{0 . 0 8 9 7}$ \\
\hline 6 & $2.43 \times 10^{-16}$ & $2.97 \times 10^{-4}$ & $3.37 \times 10^{-4}$ \\
\hline 7 & $\mathbf{0 . 0 4 3 0}$ & $\mathbf{0 . 0 4 2 6}$ & $\mathbf{0 . 0 4 3 1}$ \\
\hline 8 & $7.12 \times 10^{-17}$ & $2.94 \times 10^{-4}$ & $3.37 \times 10^{-4}$ \\
\hline 9 & $1.52 \times 10^{-16}$ & $5.04 \times 10^{-4}$ & 0.0034 \\
\hline 10 & $8.51 \times 10^{-17}$ & $3.88 \times 10^{-4}$ & $2.71 \times 10^{-4}$ \\
\hline 11 & $\mathbf{0 . 0 2 9 9}$ & $\mathbf{0 . 0 3 0 6}$ & $\mathbf{0 . 0 3 0 6}$ \\
\hline 12 & $1.30 \times 10^{-16}$ & $6.27 \times 10^{-4}$ & $8.56 \times 10^{-4}$ \\
\hline 13 & $\mathbf{0 . 0 3 3 0}$ & $\mathbf{0 . 0 3 2 9}$ & $\mathbf{0 . 0 3 2 9}$ \\
\hline 14 & $8.36 \times 10^{-17}$ & $8.96 \times 10^{-4}$ & $9.36 \times 10^{-4}$ \\
\hline 15 & $9.04 \times 10^{-17}$ & $7.43 \times 10^{-4}$ & $5.11 \times 10^{-4}$ \\
\hline \multicolumn{3}{|c}{}
\end{tabular}

Table 3. The estimated amplitudes, phase and relative errors of the test signal with no noise

\begin{tabular}{c|c|c|c|c}
\hline $\begin{array}{c}\text { Harmonic } \\
\text { order }\end{array}$ & $\begin{array}{c}\text { Amplitude } \\
\text { (p.u.) }\end{array}$ & $\begin{array}{c}e_{\mathrm{ra}} \\
(\%)\end{array}$ & $\begin{array}{c}\text { Phase } \\
(\text { degree) }\end{array}$ & $\begin{array}{c}e_{\mathrm{rp}} \\
(\%)\end{array}$ \\
\hline 1 & 0.94999 & 0.00105 & -2.36 & 12 \\
\hline 5 & 0.08999 & 0.01110 & 80.90 & 1.5 \\
\hline 7 & 0.04299 & 0.0233 & 6.94 & 12 \\
\hline 11 & 0.02996 & 0.0133 & -151.18 & 2.0 \\
\hline 13 & 0.03299 & 0.0303 & 162.92 & 0.197 \\
\hline
\end{tabular}

estimated their amplitudes correctly under the condition of no noise, 40-dB WGN and 30-dB WGN.

The estimated amplitudes and phases as well as their corresponding relative errors of the effective harmonic components contained in the original signal with no noise, 40-dB WGN and 30-dB WGN are listed in Tables 3, 4, and 5 , respectively. For the purpose of convenience, we denote the relative errors of the estimated amplitude and phase as $e_{\mathrm{ra}}$ and $e_{\mathrm{rp}}$, respectively.

It can be seen from Tables 3, 4 and 5 that the proposed method can estimate the amplitudes correctly under the condition of no noise, 40-dB WGN and 30-dB WGN, respectively. The maximum relative error is about $2 \%$, which indicates a very high accuracy. The estimation of the phases also achieves high accuracy. The relative errors of the phases are generally within $3 \%$ except for those of the fundamental frequency component and the $7^{\text {th }}$ component. Under the three noise conditions, they can sometimes reach as high as $16 \%$ because that the real phases of the fundamental frequency component and the $7^{\text {th }}$ component are -2.02 degrees and 7.9 degrees which are very small, easily leading to high relative error. But it does not influence the results of the harmonic estimation because the phase errors of the fundamental and $7^{\text {th }}$ harmonic component can reach at most 0.3232 degrees $(2.02 \times 0.16)$ and 1.264 degrees $(7.9 \times 0.16)$, respectively.
Table 4. The estimated amplitudes, phases and relative errors of the signal with 40-dB WGN

\begin{tabular}{c|c|c|c|c}
\hline $\begin{array}{c}\text { Harmonic } \\
\text { order }\end{array}$ & $\begin{array}{c}\text { Amplitude } \\
\text { (p.u.) }\end{array}$ & $\begin{array}{c}e_{\mathrm{ra}} \\
(\%)\end{array}$ & $\begin{array}{c}\text { Phase } \\
(\text { degree })\end{array}$ & $\begin{array}{c}e_{\mathrm{rp}} \\
(\%)\end{array}$ \\
\hline 1 & 0.95036 & 0.0379 & -2.22 & 9.9 \\
\hline 5 & 0.08965 & 0.389 & 81.16 & 1.1 \\
\hline 7 & 0.04261 & 0.907 & 6.70 & 15 \\
\hline 11 & 0.03062 & 2.07 & -150.83 & 2.5 \\
\hline 13 & 0.03300 & 0 & 161.52 & 0.66 \\
\hline
\end{tabular}

Table 5. The estimated amplitudes, phases and relative errors of the test signal with 30-dB WGN

\begin{tabular}{c|c|c|c|c}
\hline $\begin{array}{c}\text { Harmonic } \\
\text { order }\end{array}$ & $\begin{array}{c}\text { Amplitude } \\
\text { (p.u.) }\end{array}$ & $\begin{array}{c}e_{\text {ra }} \\
(\%)\end{array}$ & $\begin{array}{c}\text { Phase } \\
(\text { degree })\end{array}$ & $\begin{array}{c}e_{\text {rp }} \\
(\%)\end{array}$ \\
\hline 1 & 0.95010 & 0.0105 & -2.34 & 16 \\
\hline 5 & 0.09039 & 0.0403 & 79.57 & 3.1 \\
\hline 7 & 0.04261 & 0.907 & 6.85 & 13 \\
\hline 11 & 0.03014 & 0.467 & -149.80 & 1.8 \\
\hline 13 & 0.03235 & 1.97 & 160.10 & 1.5 \\
\hline
\end{tabular}

Table 6. Amplitude estimation by the proposed method, FFT and IPFFTHW (no noise)

\begin{tabular}{c|c|c|c}
\hline $\begin{array}{c}\text { Harmonic } \\
\text { order }\end{array}$ & $\begin{array}{c}\text { The proposed } \\
\text { method }\end{array}$ & FFT & IpFFTHW \\
\hline 1 & $\mathbf{0 . 9 4 9 9}$ & 0.9475 & 0.9445 \\
\hline 5 & $\mathbf{0 . 0 8 9 9}$ & 0.0897 & 0.0774 \\
\hline 7 & $\mathbf{0 . 0 4 2 9}$ & 0.0404 & 0.0401 \\
\hline 11 & $\mathbf{0 . 0 2 9 9}$ & 0.0313 & 0.0299 \\
\hline 13 & $\mathbf{0 . 0 3 2 9}$ & 0.0325 & 0.0317 \\
\hline
\end{tabular}

Table 7. Amplitude estimation by the proposed method, FFT and IpFFTHW (40-dB WGN)

\begin{tabular}{c|c|c|c}
\hline Harmonic order & The proposed method & FFT & IpFFTHW \\
\hline 1 & $\mathbf{0 . 9 5 0 4}$ & 0.9473 & 0.9445 \\
\hline 5 & $\mathbf{0 . 0 8 9 7}$ & 0.0904 & 0.0771 \\
\hline 7 & $\mathbf{0 . 0 4 2 6}$ & 0.0406 & 0.0400 \\
\hline 11 & $\mathbf{0 . 0 3 0 6}$ & 0.0321 & 0.0296 \\
\hline 13 & $\mathbf{0 . 0 3 2 9}$ & 0.0318 & 0.0323 \\
\hline
\end{tabular}

Table 8. Amplitude estimation by the proposed method, FFT and IpFFTHW (30-dB WGN)

\begin{tabular}{c|c|c|c}
\hline Harmonic order & The proposed method & FFT & IpFFTHW \\
\hline 1 & $\mathbf{0 . 9 5 0 1}$ & 0.9450 & 0.9451 \\
\hline 5 & $\mathbf{0 . 0 9 0 4}$ & 0.0917 & 0.0759 \\
\hline 7 & $\mathbf{0 . 0 4 2 6}$ & 0.0358 & 0.0394 \\
\hline 11 & $\mathbf{0 . 0 3 0 1}$ & 0.0317 & 0.0288 \\
\hline 13 & $\mathbf{0 . 0 3 2 4}$ & 0.0292 & 0.0307 \\
\hline
\end{tabular}

For the purpose of comparison, The IEC 61000-4-7 recommended method [17] FFT and an Interpolated FFT with the Hanning Window (IpFFTHW) [18] have been employed to estimate the amplitudes of the harmonics contained in the test signal.

The estimation results of the three methods under condition of different noise levels are presented in Tables 6, 7,8 , and the best results among the three methods have been highlighted in bold. It can be seen from these tables that in most cases, the proposed method obtains better 


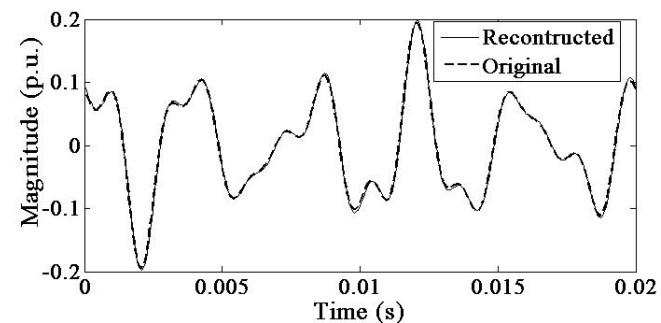

(a)

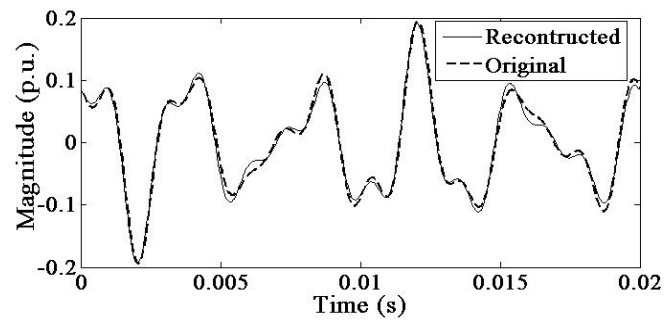

(b)

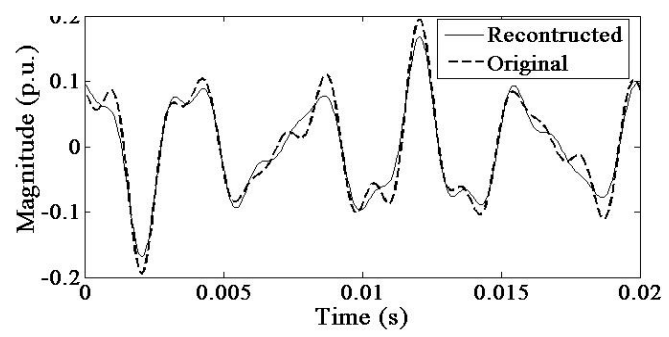

(c)

Fig. 8. Actual (dotted) and reconstructed waveforms for all the harmonics except the fundamental component. (a) 30-dB WGN. (b) 20-dB WGN. (c) 10-dB WGN.

results in the estimation of the amplitudes. In the no-noise scenario, the performance of FFT and IpFFTHW is a little worse than the proposed method. While with the 40-dB and $30-\mathrm{dB}$ WGN added in the original signal, respectively, the accuracy of FFT and IpFFTHW is remarkably degraded. On the contrary, the proposed method maintains its high accuracy despite of the presence of WGN.

In order to evaluate the performance of the proposed method graphically, Fig. 8 depicts the actual waveform of the harmonic components except the fundamental frequency component and the reconstructed waveform of the same harmonics using the estimated amplitudes and phases. It can be seen from Fig. 8 that the estimated and the actual signals are essentially identical under the condition of 30 $\mathrm{dB}$ WGN. With the noise getting heavier, the difference between the estimated and the actual signals begins to get obvious, but the estimated waveforms still maintain the approximate shape even when the SNR is getting as low as $10 \mathrm{~dB}$.

\subsection{Simulation results with the fundamental frequency deviation}

In this section, the accuracy of the proposed method and

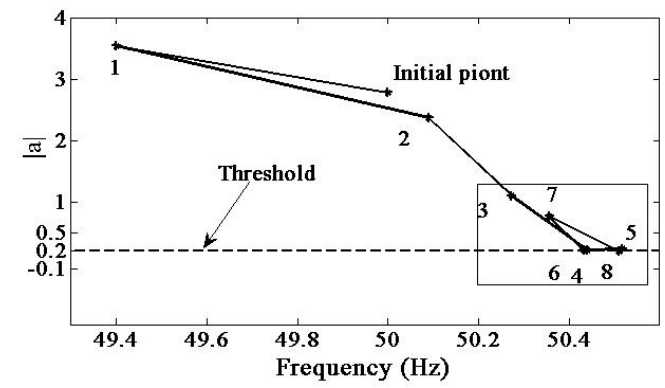

(a)

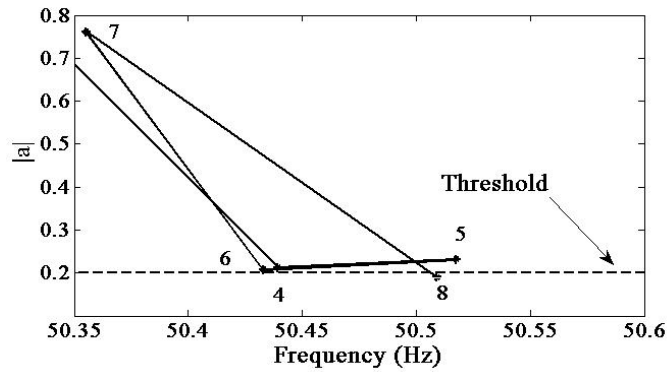

(b)

Fig. 9. The curve of the adjusting steps with the deviated frequency $50.5 \mathrm{~Hz}$. (a) All steps. (b) The details of the last several steps.

IpFFTHW under non-synchronous sampling, i.e. the fundamental frequency deviates from its nominal value, is evaluated.

\subsubsection{Amplitude estimation under fundamental frequency deviation}

In this subsection, we adopt the same simulation model as used in subsection 4.3 and the harmonic contents are the same as those in subsection 4.3. We set two fundamental frequency deviation scenarios: a positive deviation and a negative deviation. In the first case, the deviated fundamental frequency is assumed to be $50.5 \mathrm{~Hz}$ while the rated fundamental frequency in this paper is set as $50 \mathrm{~Hz}$. To make the simulation more practical, $30-\mathrm{dB}$ WGN is added to the original test signal. The frequency estimation is conducted according to Fig. 3 and all the steps (in this case, 8 frequency adjustments in total) are shown as Fig. 9.

In Fig. 9 (a), every star * means the adjusted frequency and the corresponding $|a|$ at each step. And the number near each star * means the order of each adjusting step. To clearly illustrate the last several steps (i.e., in the rectangle in Fig. 9 (a)), we plot Fig. 9 (b) to show the details of them. Through 8 steps, the final estimated frequency is $50.5090 \mathrm{~Hz}$, which is quite close to the real deviated frequency $50.5 \mathrm{~Hz}$.

Table 9 lists the amplitude estimation results and their errors (\%) using Park transform with and without frequency adjustment, and the IpFFTHW. And the better results have been highlighted in bold. (.)/(.) means "the estimated amplitude/error (\%)". 
Table 9. The amplitude estimation results with and without frequency adjustment

\begin{tabular}{c|c|c|c}
\hline $\begin{array}{c}\text { Harmonic } \\
\text { order }\end{array}$ & $\begin{array}{c}\text { With frequency } \\
\text { adjustment }\end{array}$ & $\begin{array}{c}\text { Without frequency } \\
\text { adjustment }\end{array}$ & IpFFTHW \\
\hline 1 & $\mathbf{0 . 9 5 0 3 / 0 . 0 3}$ & $0.9507 / 0.07$ & $0.9398 / 1.07$ \\
\hline 5 & $\mathbf{0 . 0 9 0 8 / 0 . 8 8}$ & $0.0881 / 2.11$ & $0.0842 / 6.44$ \\
\hline 7 & $0.0445 / 3.49$ & $0.0409 / 4.88$ & $\mathbf{0 . 0 4 2 6 / 0 . 9 3}$ \\
\hline 11 & $\mathbf{0 . 0 3 0 3 / 1 . 0 0}$ & $0.0313 / 4.33$ & $0.0260 / 13.30$ \\
\hline 13 & $\mathbf{0 . 0 3 2 8} / \mathbf{0 . 6 1}$ & $0.0311 / 5.76$ & $0.0275 / 16.67$ \\
\hline
\end{tabular}

Table 10. The amplitude estimation results with and without frequency adjustment

\begin{tabular}{c|c|c|c}
\hline $\begin{array}{c}\text { Harmonic } \\
\text { order }\end{array}$ & $\begin{array}{c}\text { With frequency } \\
\text { adjustment }\end{array}$ & $\begin{array}{c}\text { Without frequency } \\
\text { adjustment }\end{array}$ & IpFFTHW \\
\hline 1 & $\mathbf{0 . 9 5 0 5 / 0 . 0 5}$ & $0.9512 / 0.13$ & $0.9312 / 1.98$ \\
\hline 5 & $\mathbf{0 . 0 9 1 0 / 1 . 1 1}$ & $0.0875 / 2.78$ & $0.0749 / 16.78$ \\
\hline 7 & $0.0445 / 3.49$ & $\mathbf{0 . 0 4 3 1 / 0 . 2 4}$ & $0.0352 / 18.14$ \\
\hline 11 & $\mathbf{0 . 0 3 0 0 / 0}$ & $0.0299 / 0.33$ & $0.0286 / 4.67$ \\
\hline 13 & $\mathbf{0 . 0 3 4 3 / 3 . 9 4}$ & $0.0309 / 6.36$ & $0.0313 / 5.15$ \\
\hline
\end{tabular}

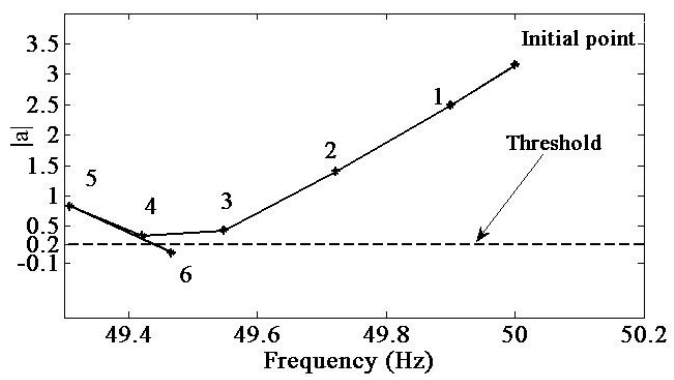

Fig. 10. The curve of the adjusting steps with the deviated frequency $49.5 \mathrm{~Hz}$.

From Table 9, it can be seen that the frequency adjustment processing has improved the accuracy of the proposed method greatly, especially in the estimation of the high-order harmonic component. And in most cases, the proposed method achieves higher accuracy than IpFFTHW.

In the second case, the deviated frequency is $49.5 \mathrm{~Hz}$ and the same processing has been done in this case. Fig. 10 and Table 10 present the results.

From Fig. 10, it can be seen that through 6 steps, the final estimated frequency is about $49.4666 \mathrm{~Hz}$, which is considerably close to the real frequency $49.5 \mathrm{~Hz}$. Similar to the first case, Table 10 shows that in most cases, the performance of the proposed method has been slightly improved by adjusting the fundamental frequency before estimating the amplitudes of the harmonic components. And the proposed method can obviously obtain better results than IpFFTHW.

\subsubsection{Frequency estimation under frequency deviation}

In addition to the comparison of the amplitude estimation, we also compare the frequency estimation results between the proposed method and IpFFTHW. The harmonic components of the test signal is the same as 4.4.1, but the fundamental frequency is set from $49.3 \mathrm{~Hz}$ to 50.8 at the
Table 11. The frequency estimation results using the proposed method and IpFFTHW

\begin{tabular}{c|c|c|c}
\hline$f_{\text {real }}(\mathrm{Hz})$ & 49.3 & 49.6 & 49.9 \\
\hline$f_{\text {pro }}(\mathrm{Hz}) /$ error $(\%)$ & $\mathbf{4 9 . 2 7 / 0 . 0 6}$ & $\mathbf{4 9 . 6 0 / 0}$ & $49.85 / 0.10$ \\
\hline$f_{\text {lpFFTHW }}(\mathrm{Hz}) /$ error $(\%)$ & $49.26 / 0.08$ & $49.58 / 0.04$ & $\mathbf{4 9 . 8 9 / 0 . 0 2}$ \\
\hline$f_{\text {real }}(\mathrm{Hz})$ & 50.2 & 50.5 & 50.8 \\
\hline$f_{\text {pro }}(\mathrm{Hz}) /$ error $(\%)$ & $\mathbf{5 0 . 1 8 / 0 . 0 4}$ & $\mathbf{5 0 . 5 1 / 0 . 0 2}$ & $50.77 / 0.06$ \\
\hline$f_{\text {lpFFTHW }}(\mathrm{Hz}) /$ error $(\%)$ & $\mathbf{5 0 . 1 8 / 0 . 0 4}$ & $50.48 / 0.04$ & $\mathbf{5 0 . 8 1 / 0 . 0 2}$ \\
\hline
\end{tabular}

step of $0.3 \mathrm{~Hz}$. The simulation results is listed in Table 11 , where $f_{\text {real }}$ stands for the real deviated fundamental frequency, $f_{\text {pro }}$ stands for the frequency estimation by the proposed method, $f_{\mathrm{IPFFT}}$ stands for the frequency estimation by IpFFTHW.

From the simulation results above, it can be seen that both method achieve high accuracy in frequency estimation. Moreover, in most cases, the performance of the proposed method is better than that of IpFFTHW.

\subsection{The effect of inter-harmonic on the performance of the proposed method}

\subsubsection{The effect of single inter-harmonic on the perfor- mance of the proposed method}

To evaluate the performance of the proposed method in the presence of inter-harmonics, an inter-harmonic is added to the original signal described in section 4.1. The parameters of the inter-harmonic is set the same as [3], i.e, the frequency, the amplitude and the phase of the interharmonic are set to be $20 \mathrm{~Hz}, 0.505$ p.u., 75.6 degrees, respectively.

To make the simulation more practical, $30-\mathrm{dB}$ WGN is added to the signal above. And the results of the amplitude estimation of integral harmonic under two scenarios (with inter-harmonic and without inter-harmonic) are presented in Table 12, where (.)/(.) means "the estimated amplitude (p.u.)/ error (\%)".

Table 12. The amplitude estimation results with and without single inter-harmonic

\begin{tabular}{c|c|c}
\hline Harmonic order & Without inter-harmonic & With inter-harmonic \\
\hline 1 & $0.9501 / 0.01$ & $0.8846 / 6.88$ \\
\hline 5 & $0.0904 / 0.44$ & $0.0891 / 1.00$ \\
\hline 7 & $0.0426 / 0.93$ & $0.0447 / 3.95$ \\
\hline 11 & $0.0301 / 0.33$ & $0.0308 / 2.67$ \\
\hline 13 & $0.0324 / 1.82$ & $0.0333 / 0.91$ \\
\hline
\end{tabular}

It can be seen from the table above that the interharmonic has decreased the accuracy of the proposed method to some extent. The maximum estimation error is $6.88 \%$, which is acceptable in practice.

\subsubsection{The effect of mixed inter-harmonics on the perfor- mance of the proposed method}

Considering that there are usually more than one inter- 
Table 13. The amplitude estimation results with and without mixed inter-harmonics

\begin{tabular}{c|c|c}
\hline Harmonic order & Without inter-harmonic & With inter-harmonic \\
\hline 1 & $0.9501 / 0.01$ & $0.9640 / 1.48$ \\
\hline 5 & $0.0904 / 0.44$ & $0.0884 / 1.78$ \\
\hline 7 & $0.0426 / 0.93$ & $0.0421 / 2.09$ \\
\hline 11 & $0.0301 / 0.33$ & $0.0319 / 6.33$ \\
\hline 13 & $0.0324 / 1.82$ & $0.0312 / 5.45$ \\
\hline
\end{tabular}

harmonic in the power electrical signal [19], in this subsection we check the performance in the presence of mixed inter-harmonics. We add another inter-harmonic in the original signal described in section 4.1, in addition to the $20-\mathrm{Hz}$ inter-harmonic. The frequency, amplitude and phase of this inter-harmonic are set to be $75 \mathrm{~Hz}, 0.5$ p.u., 30 degrees, respectively. Similarly, to make the simulation more practical, 30-dB WGN is added to the signal above. Table 13 lists the simulation results.

It can be seen from Table 13 that the performance of the proposed method is not deteriorated by the mixed interharmonics dramatically. The maximum error in this case is $6.33 \%$, which meets the standard in practice.

\section{Validation Based on a Popular Case}

To further illustrate the effectiveness of the proposed method, a popular case originally created at the Manitoba HVDC Research Centre by Dr. Reformat in Manitoba, Canada and adopted in [20] later, is established in PSCAD/ EMTDC to generate test signals. This case is modelled as shown in Fig. 11, where an active filter is connected through a $20 \mathrm{kVA}, \mathrm{Y}-\mathrm{Y}$ transformer to a $200 \mathrm{~V}, 50 \mathrm{~Hz}$, three-phase power source, with a 6-pulse converter load at the end.

The test signal is phase-a's the line current in the primary winding of the transformer in Fig. 11 and its waveform is plotted in Fig. 12.

After applying the proposed method to estimate the harmonics of the test signal, we reconstruct the waveform using the estimated amplitudes and phases. The reconstructed waveform in the first fundamental cycle is compared with the original waveform in Fig. 13. It is apparent from Fig. 13 that the estimated waveform of the test signal (the solid

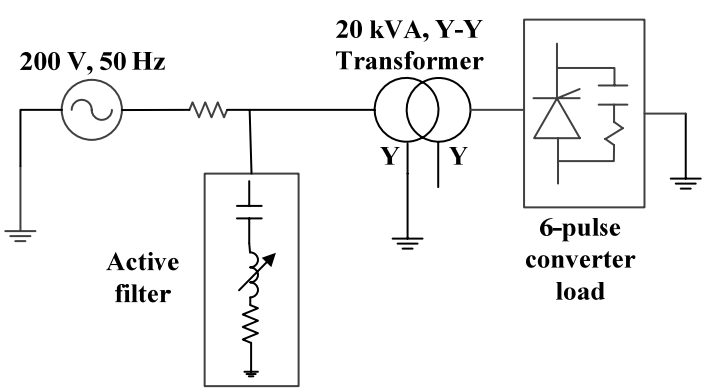

Fig. 11. The single line diagram of a popular case

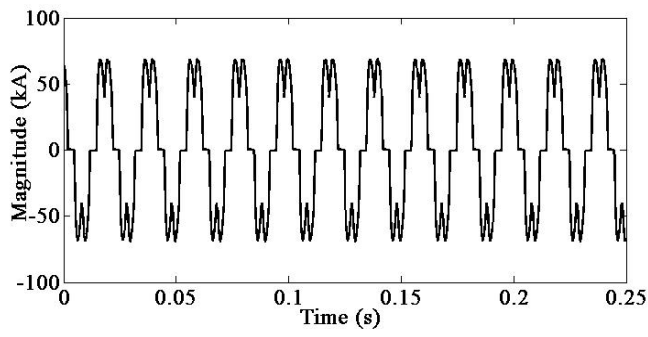

Fig. 12. The waveform of the test signal

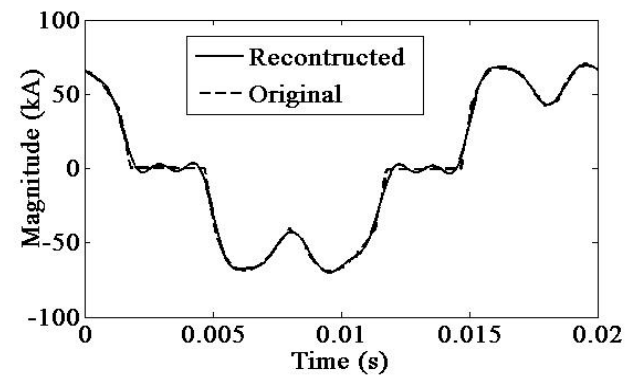

Fig. 13. A comparison of the reconstructed waveform with the original waveform.

line) is almost identical with that of the original signal. The difference between the original and reconstructed waveforms mainly results from the harmonic parameter estimation errors and the missing harmonic components.

\section{Conclusion}

This paper has presented a new algorithm for the power harmonic estimation. This algorithm employs Park transform to estimate the amplitudes, phases of the harmonics. Besides, the harmonic estimation in the scenario of frequency deviation is also considered. A typical simulation model widely adopted is established using MATLAB for simulation studies. Simulation results have demonstrated that the proposed method achieves higher accuracy than FFT and IpFFTHW in amplitude estimation; in the frequency deviation scenario, the proposed method achieves higher accuracy in fundamental frequency and amplitude estimation; the presence of the inter-harmonics cannot deteriorate the performance of the proposed method dramatically. At last, a popular case is used for further validation. Thus, the proposed method can provide an alternative way to harmonic analysis, especially in the frequency deviation scenario.

\section{Appendix A}

The goal is to prove:

$$
\left\{\begin{array}{l}
S_{\mathrm{dn}}^{\prime}=S_{\mathrm{dn}}(t) \\
S_{\mathrm{qn}}^{\prime}=S_{\mathrm{qn}}(t)
\end{array}\right.
$$


Compare (21) with (24), it can be easily derived that proving (22) is equivalent to prove:

$$
\left\{\begin{array}{l}
\frac{1}{N} \sum_{l=1}^{N} S_{\mathrm{doth}}(l)=0 \\
\frac{1}{N} \sum_{l=1}^{N} S_{\mathrm{qoth}}(l)=0
\end{array}\right.
$$

Considering $S_{\text {oth }}(t)$ can be viewed as the summation of all the harmonic components except the $n^{\text {th }}$ harmonic component as (16) shows, according to the linear property of the Park transform, if we can prove:

$$
\left\{\begin{array}{l}
\frac{1}{N} \sum_{l=1}^{N} S_{\mathrm{dk}}(l)=0 \\
\frac{1}{N} \sum_{l=1}^{N} S_{\mathrm{qk}}(l)=0 \quad(k=1,2 \ldots m \text { and } k \neq n)
\end{array}\right.
$$

then we can prove (25) consequently. Once (25) has been proven, as mentioned above, (24) can be proven as a result. Therefore, the main problem needing to be tackled for proving (24) is to prove (26). Next, we will present how to prove (26).

In circuit theory, the time-domain form of a group of signals with the same frequency can be expressed as a group of phasors and vice versa. For instance, assume three signals with the same frequency of $50 \mathrm{~Hz}$ are given as:

$$
\left\{\begin{array}{l}
s_{1}(t)=20 \sin \left(100 \pi t+\frac{\pi}{2}\right) \\
s_{2}(t)=30 \sin \left(100 \pi t+\frac{\pi}{3}\right) \\
s_{3}(t)=40 \sin \left(100 \pi t+\frac{\pi}{4}\right)
\end{array}\right.
$$

These signals can be expressed in the form of phasors as $\dot{s}_{1}=20 \angle \pi / 2, \dot{s}_{2}=30 \angle \pi / 3$ and $\dot{s}_{3}=40 \angle \pi / 4$, respectively, where $\dot{s}_{1}, \dot{s}_{1}, \dot{s}_{1}$ denote the phasors of $s_{1}(t)$, $s_{2}(t)$ and $s_{3}(t)$, respectively.

Similarly, the $k^{\text {th }}$ harmonic component in the three-phase signal in (16) can be expressed in the form of phasors as:

$$
\dot{S}_{\mathrm{k}}=\left[\begin{array}{c}
\dot{S}_{\mathrm{ka}} \\
\dot{S}_{\mathrm{kb}} \\
\dot{S}_{\mathrm{kc}}
\end{array}\right]=\left[\begin{array}{c}
A_{k} \angle \varphi_{k} \\
A_{k} \angle\left(\varphi_{k}-2 k \pi / 3 n\right) \\
A_{k} \angle\left(\varphi_{k}-4 k \pi / 3 n\right)
\end{array}\right]
$$

where $\dot{S}_{\mathrm{k}}$ represents the phasor vector of the three-phase signal, $\dot{S}_{\mathrm{ka}}, \dot{S}_{\mathrm{kb}}$ and $\dot{S}_{\mathrm{kc}}$ represent the phasors of $S_{\mathrm{ka}}(t), S_{\mathrm{kb}}(t)$ and $S_{\mathrm{kc}}(t)$, respectively. Noticing that $k \neq n$, the three phasors in (28) are not three-phase symmetric. In order to analyze the three-phase asymmetric phasors, a symmetric component analysis method [21] is introduced in this paper. This method can decompose any group of three three-phase asymmetric phasors into three groups of symmetric phasors (i.e., the positive-sequence phasors, the negative-sequence phasors and the zerosequence phasors) via a symmetric component transform matrix:

$$
T=\frac{1}{3}\left[\begin{array}{ccc}
1 & a & a^{2} \\
1 & a^{2} & a \\
1 & 1 & 1
\end{array}\right]
$$

where $a=1 \angle 2 \pi / 3$ is the rotation operator. Then the positive-sequence phasor, negative-sequence phasor and zero-sequence phasor of phase a, denoted as $\dot{S}_{\mathrm{a} 1}, \dot{S}_{\mathrm{a} 2}$ and $\dot{S}_{\mathrm{a} 0}$, respectively, can be obtained as follows:

$$
\left[\begin{array}{c}
\dot{S}_{\mathrm{a} 1} \\
\dot{S}_{\mathrm{a} 2} \\
\dot{S}_{\mathrm{a} 0}
\end{array}\right]=T \dot{S}_{k}=\frac{1}{3}\left[\begin{array}{ccc}
1 & a & a^{2} \\
1 & a^{2} & a \\
1 & 1 & 1
\end{array}\right]\left[\begin{array}{c}
\dot{S}_{\mathrm{ka}} \\
\dot{S}_{\mathrm{kb}} \\
\dot{S}_{\mathrm{kc}}
\end{array}\right]
$$

And the positive-sequence, negative-sequence and zerosequence phasors of phase $\mathrm{b}$ and phase $\mathrm{c}$ can be obtained as follows:

$$
\left\{\begin{array}{cl}
\dot{S}_{\mathrm{b} 1}=a^{2} \dot{S}_{\mathrm{a} 1}, & \dot{S}_{\mathrm{c} 1}=a \dot{S}_{\mathrm{a} 1} \\
\dot{S}_{\mathrm{b} 2}=a \dot{S}_{\mathrm{a} 2}, & \dot{S}_{\mathrm{c} 2}=a^{2} \dot{S}_{\mathrm{a} 2} \\
\dot{S}_{\mathrm{b} 0}=\dot{S}_{\mathrm{a} 0}, & \dot{S}_{\mathrm{c} 0}=\dot{S}_{\mathrm{a} 0}
\end{array}\right.
$$

Then the $k^{\text {th }}$ harmonic component $\dot{S}_{\mathrm{k}}$ in (28) can be expressed as:

$$
\dot{S}_{\mathrm{k}}=\dot{S}_{\mathrm{k} 1}+\dot{S}_{\mathrm{k} 2}+\dot{S}_{\mathrm{k} 0}
$$

where

$$
\dot{S}_{\mathrm{k} 1}=\left[\begin{array}{c}
\dot{S}_{\mathrm{a} 1} \\
\dot{S}_{\mathrm{b} 1} \\
\dot{S}_{\mathrm{c} 1}
\end{array}\right], \dot{S}_{\mathrm{k} 2}=\left[\begin{array}{c}
\dot{S}_{\mathrm{a} 2} \\
\dot{S}_{\mathrm{b} 2} \\
\dot{S}_{\mathrm{c} 2}
\end{array}\right], \dot{S}_{\mathrm{k} 0}=\left[\begin{array}{c}
\dot{S}_{\mathrm{a} 0} \\
\dot{S}_{\mathrm{b} 0} \\
\dot{S}_{\mathrm{c} 0}
\end{array}\right]
$$

Thus, according to its linear property, the Park transform of $S_{\mathrm{k}}(t)$ equals the summation of the Park transforms of $S_{\mathrm{k} 1}(t), S_{\mathrm{k} 2}(t)$ and $S_{\mathrm{k} 0}(t)$. Next, we will discuss the Park transforms of $S_{\mathrm{k} 1}(t), S_{\mathrm{k} 2}(t)$ and $S_{\mathrm{k} 0}(t)$, respectively.

The group of positive-sequence signals $S_{\mathrm{k} 1}(t)$ can be expressed as:

$$
S_{\mathrm{k} 1}(t)=\left[\begin{array}{c}
S_{\mathrm{a} 1}(t) \\
S_{\mathrm{b} 1}(t) \\
S_{\mathrm{c} 1}(t)
\end{array}\right]=\left[\begin{array}{c}
A_{\mathrm{k} 1} \cos \left(k \omega_{1} t+\phi_{k 1}\right) \\
A_{\mathrm{k} 1} \cos \left(k \omega_{1} t+\phi_{k 1}-2 \pi / 3\right) \\
A_{\mathrm{k} 1} \cos \left(k \omega_{1} t+\phi_{k 1}-4 \pi / 3\right)
\end{array}\right]
$$

where, $A_{\mathrm{k} 1}$ and $\phi_{\mathrm{k} 1}$ denote the amplitude and phase of 
the positive component of the $k^{\text {th }}$ harmonic component. The Park transform of $S_{\mathrm{k} 1}(t)$ via the Park matrix in (13) can be calculated as follows:

$$
\left\{\begin{array}{l}
S_{1 \mathrm{~d}}(t)=A_{\mathrm{k} 1} \cos \left[-\phi_{k}+(n-k) \omega_{1} t\right] \\
S_{1 \mathrm{q}}(t)=A_{\mathrm{k} 1} \sin \left[-\phi_{k}+(n-k) \omega_{1} t\right] \\
S_{10}(t)=0
\end{array}\right.
$$

It can be easily seen that $S_{1 \mathrm{~d}}(t)$ and $S_{1 \mathrm{q}}(t)$ are both sinusoidal signals whose frequency is integer multiples of the fundamental frequency. Thus the average value of the samples in one fundamental cycle of $S_{1 \mathrm{~d}}(t)$ or $S_{1 \mathrm{q}}(t)$ is obviously zero, i.e.:

$$
\left\{\begin{array}{l}
\frac{1}{N} \sum_{l=1}^{N} S_{1 \mathrm{~d}}(l)=0 \\
\frac{1}{N} \sum_{l=1}^{N} S_{1 \mathrm{q}}(l)=0
\end{array}\right.
$$

Similarly, the group of negative-sequence signals $S_{\mathrm{k} 2}(t)$ can be expressed as:

$$
S_{\mathrm{k} 2}(t)=\left[\begin{array}{c}
S_{\mathrm{a} 2}(t) \\
S_{\mathrm{b} 2}(t) \\
S_{\mathrm{c} 2}(t)
\end{array}\right]=\left[\begin{array}{c}
A_{\mathrm{k} 2} \cos \left(k \omega_{1} t+\phi_{k 2}\right) \\
A_{\mathrm{k} 2} \cos \left(k \omega_{1} t+\phi_{k 2}+2 \pi / 3\right) \\
A_{\mathrm{k} 2} \cos \left(k \omega_{1} t+\phi_{k 2}-2 \pi / 3\right)
\end{array}\right]
$$

Then the Park transform of $S_{\mathrm{k} 2}(t)$ by the Park matrix in (13) can be calculated as follows:

$$
\left\{\begin{array}{l}
S_{2 \mathrm{~d}}(t)=A_{\mathrm{k} 2} \cos \left[-\phi_{\mathrm{k} 2}+(n+k) \omega_{1} t\right] \\
S_{2 \mathrm{q}}(t)=A_{\mathrm{k} 2} \sin \left[-\phi_{\mathrm{k} 2}+(n+k) \omega_{1} t\right] \\
S_{20}(t)=0
\end{array}\right.
$$

Similarly, the average value of the samples in one fundamental cycle of $S_{2 \mathrm{~d}}(t)$ or $S_{2 \mathrm{q}}(t)$ is obviously zero, i.e.:

$$
\left\{\begin{array}{l}
\frac{1}{N} \sum_{l=1}^{N} S_{2 \mathrm{~d}}(l)=0 \\
\frac{1}{N} \sum_{l=1}^{N} S_{2 \mathrm{q}}(l)=0
\end{array}\right.
$$

The group of zero-sequence signals $S_{\mathrm{k} 0}(t)$ can be expressed as:

$$
S_{\mathrm{k} 0}(t)=\left[\begin{array}{c}
S_{\mathrm{a} 0}(t) \\
S_{\mathrm{b} 0}(t) \\
S_{\mathrm{c} 0}(t)
\end{array}\right]=\left[\begin{array}{c}
A_{\mathrm{k} 0} \cos \left(k \omega_{1} t+\phi_{k 0}\right) \\
A_{\mathrm{k} 0} \cos \left(k \omega_{1} t+\phi_{k 0}\right) \\
A_{\mathrm{k} 0} \cos \left(k \omega_{1} t+\phi_{k 0}\right)
\end{array}\right]
$$

The Park transform of $S_{\mathrm{k} 0}(t)$ can be easily derived as:

$$
\left\{\begin{array}{l}
S_{0 \mathrm{~d}}(t)=0 \\
S_{0 \mathrm{q}}(t)=0 \\
S_{00}(t)=0
\end{array}\right.
$$

Thus the average value of the samples in one fundamental cycle of $S_{0 \mathrm{~d}}(t)$ or $S_{0 \mathrm{q}}(t)$ is obviously zero:

$$
\left\{\begin{array}{l}
\frac{1}{N} \sum_{l=1}^{N} S_{0 \mathrm{~d}}(l)=0 \\
\frac{1}{N} \sum_{l=1}^{N} S_{0 \mathrm{q}}(l)=0
\end{array}\right.
$$

Therefore, according to (36), (39) and (42), (26) can be proven. As mentioned above, (24) can be proved consequently.

\section{Appendix B}

When no frequency deviation happens, setting $k^{\prime}$ in (9) to be 1 , the transformed d-axis component of the fundamental frequency component can be obtained as follows:

$$
S_{\mathrm{d}}(t)=\sum_{k=2}^{m} A_{k} \cos \left[-\varphi_{k}+(1-k) \omega_{1} t\right]+A_{1} \cos \left(-\varphi_{1}\right)
$$

Considering that $S_{\mathrm{d}}(t)$ is periodic, we just use the samples of $S_{\mathrm{d}}(t)$ in the first cycle to calculate the values of $a$ and $b$ in the fitting function $y=a x+b$. According to the linear fitting theory [22, 23], $a$ and $b$ can be calculated as:

$$
\left\{\begin{array}{c}
b=\bar{y}-a \bar{x} \\
a=\frac{\overline{x y}-\bar{x} \cdot \bar{y}}{\overline{x^{2}}-\bar{x}^{2}}
\end{array}\right.
$$

Assuming that there are $N$ samples in one fundamental cycle, then the $N$ samples can be discretized as Table 14.

Table 14. The discretized sequence of $S_{\mathrm{d}}(t)-t$

\begin{tabular}{c|c|c|c}
\hline$S_{\mathrm{d}}(t)(y)$ & $S_{\mathrm{d}}(\Delta t)$ & $\cdots$ & $S_{\mathrm{d}}(T)$ \\
\hline$t(x)$ & $\Delta t$ & $\ldots$ & $T$ \\
\hline
\end{tabular}

where $T$ is the fundamental cycle, $\Delta t=T / N$ is the sampling interval.

$\bar{x}, \bar{y}$ and $\overline{x^{2}}$ can be easily calculated as:

$$
\left\{\begin{array}{l}
\bar{x}=\frac{(N+1) \Delta t}{2} \\
\bar{y}=A_{1} \cos \left(-\varphi_{1}\right) \\
\overline{x^{2}}=\frac{(N+1)(2 N+1) \Delta t^{2}}{6}
\end{array}\right.
$$


Next calculate $\overline{x y}$ :

$$
\begin{aligned}
\overline{x y} & =\frac{1}{N} \sum_{l=1}^{N}\left\{\sum_{k=2}^{m} A_{k} \cos \left[-\varphi_{k}+(1-k) \omega_{1}(l \Delta t)\right]\right. \\
& \left.+A_{1} \cos \left(-\varphi_{1}\right)\right\} \cdot(l \Delta t) \\
= & \frac{1}{N} \sum_{l=1}^{N} A_{1} \cos \left(-\varphi_{1}\right) \cdot(l \Delta t)+\sum_{k=2}^{m}\left\{\frac{1}{N} \sum_{l=1}^{N} A_{k}\right. \\
& \left.\cos \left[-\varphi_{k}+(1-k) \omega_{1}(l \Delta t)\right] \cdot(l \Delta t)\right\} \\
= & \frac{A_{1} \cos \left(-\varphi_{1}\right)(N+1) \Delta t}{2}+\sum_{k=2}^{m}\left\{\frac{1}{N} \frac{1}{\Delta t} \sum_{l=1}^{N} A_{k}\right. \\
& \left.\cos \left[-\varphi_{k}+(1-k) \omega_{1}(l \Delta t) \cdot\right](l \Delta t) \cdot \Delta t\right\}
\end{aligned}
$$

Noticing that $\sum_{l=1}^{N} A_{k} \cos \left[-\varphi_{k}+(1-k) \omega_{1}(l \Delta t) \cdot\right](l \Delta t) \cdot \Delta t$ is the discrete form of the integral

$\int_{0}^{T} A_{k} \cos \left[-\varphi_{1}+(1-k) \omega_{1} t\right] \cdot t \mathrm{~d} t$ which can be calculated as:

$$
\begin{aligned}
& \int_{0}^{T} A_{k} \cos \left[-\varphi_{k}+(1-k) \omega_{1} t\right] \cdot t \mathrm{~d} t \\
= & \left.\frac{\sin \left[-\varphi_{k}+(1-k) \omega_{1} t\right] \cdot A_{k} t}{(1-k) \omega_{1}}\right|_{0} ^{T} \\
& -\int_{0}^{T} \frac{A_{k}}{(1-k) \omega_{1}} \sin \left[-\varphi_{k}+(1-k) \omega_{1} t\right] \mathrm{d} t \\
= & \left.\frac{\sin \left[-\varphi_{k}+(1-k) \omega_{1} t\right] \cdot A_{k} t}{(1-k) \omega_{1}}\right|_{0} ^{T} \\
= & \frac{\sin \left(-\varphi_{k}\right) \cdot A_{k} T}{(1-k) \omega_{1}} \\
= & \frac{\sin \left(-\varphi_{k}\right) \cdot A_{k}}{(1-k) \cdot 2 \pi \cdot\left(f_{1}\right)^{2}}
\end{aligned}
$$

Therefore

$$
\begin{aligned}
& \sum_{k=2}^{m}\left\{\frac{1}{N} \sum_{l=1}^{N} A_{k}\right. \\
& \left.\quad \cos \left[-\varphi_{k}+(1-k) \omega_{1}(l \Delta t)\right] \cdot(l \Delta t)\right\} \\
& =\sum_{k=2}^{m} \frac{\sin \left(-\varphi_{k}\right) \cdot A_{k}}{(1-k) \cdot 2 \pi \cdot\left(f_{1}\right)^{2} \cdot N \Delta t} \\
& =\sum_{k=2}^{m} \frac{\sin \left(-\varphi_{k}\right) \cdot A_{k}}{(1-k) \cdot 2 \pi \cdot f_{1}}
\end{aligned}
$$

Considering $\left|\sin \left(-\varphi_{k}\right)\right| \leq 1, A_{k} \leq 1$, thus:

$$
\left|\sin \left(-\varphi_{k}\right) \cdot A_{k}\right| \ll\left|(1-k) \cdot 2 \pi \cdot f_{1}\right|
$$

Therefore,

$$
\sum_{k=2}^{m} \frac{\sin \left(-\varphi_{k}\right) \cdot A_{k}}{(1-k) \cdot 2 \pi \cdot f_{1}} \approx 0
$$

Consequently,

$$
\overline{x y}=\frac{A_{1} \cos \left(-\varphi_{1}\right)(N+1) \Delta t}{2}
$$

Substituting (45) and (49) to (44), $a$ can be obtained from:

$$
\begin{aligned}
& a=\bar{y}-\frac{\overline{x y}-\bar{x} \cdot \bar{y}}{\overline{x^{2}}-\bar{x}^{2}} \\
& \approx \frac{\frac{A_{1} \cos \left(-\varphi_{1}\right)(N+1) \Delta t}{2}-\frac{(N+1) \Delta t}{2} \cdot A_{1} \cos \left(-\varphi_{1}\right)}{\bar{x}^{2}-\bar{x}^{2}}=0
\end{aligned}
$$

When $|a|$ calculated does not equal zero, it can be concluded that frequency deviation happens.

\section{Acknowledgements}

The work was supported by Guangdong Innovative Research Team Program (No. 201001N0104744201) and National Natural Science Foundation of China (No. 51207058).

\section{References}

[1] Ren. J., and M. Kezunovic, "A wavelet method for power system frequency and harmonic estimation," in North American Power Symposium (NAPS). pp. 16, 2010.

[2] J. Z. Yang, C. S. Yu, and C. W. Liu, "A new method for power signal harmonic analysis," IEEE Trans. Power Del., Vol. 20, No.2, pp. 1235-1239, Apr. 2005.

[3] Z. Lu, T. Y. Ji, W. H. Tang, and Q. H. Wu, "Optimal harmonic estimation using a particle swarm optimizer," IEEE Trans. Power Del., Vol. 23, No. 2, pp. 11661174, Apr. 2008.

[4] Y. N. Fei, Z. Lu, W. H. Tang, and Q. H. Wu, "Harmonic estimation using a global search optimizer," Applications of Evolutionary Computing., pp. 261270, 2007.

[5] J. J. Yao, D. Di, G. Jiang, and S. Gao, "Real-Time Acceleration Harmonics Estimation for an ElectroHydraulic Servo Shaking Table Using Kalman Filter With a Linear Model," IEEE Trans. Control Syst. Technol., Vol. 22, No. 2, pp. 794-800, Mar. 2014.

[6] C. I. Chen, G. W. Chang, and R. C. Hong, "Extended real model of Kalman filter for time-varying harmonics estimation," IEEE Trans. Power Del., Vol. 25, No. 1, pp. 17-26, Jan. 2010.

[7] S. Mishra, "A hybrid least square-fuzzy bacterial foraging strategy for harmonic estimation," IEEE Trans. Evol. Comput. Vol. 9, No. 1, pp. 61-73, Feb. 2005.

[8] M. Bettayeb, and U. Qidwai, "A hybrid least squaresGA-based algorithm for harmonic estimation." IEEE Trans. Power Del., Vol. 18, No. 2, pp. 377-382, Apr. 2003. 
[9] D. P. Kothari, and I. J. Nagrath, Modern power system analysis, Tata McGraw-Hill Education, chap. 2-5, 2003.

[10] R. H. Park, "Two-reaction theory of synchronous machines generalized method of analysis-part I," American Institute of Electrical Engineers, Transactions of the, Vol. 48, No. 3, pp. 716-727, Jul. 1929.

[11] J. J. Liu, J. Yang, and Z. A. Wang, "A new approach for single-phase harmonic current detecting and its application in a hybrid active power filter," in Proc. IECON'99 Proceedings. The 25th Annual Conference of the IEEE., pp. 849-854, 1999.

[12] T. Lin, M. Tsuji, and E. Yamada, "A wavelet approach to real time estimation of power system frequency," in Proc. The 40th SICE Annual Conference, pp. 5865, 2001.

[13] De Carvalho, and Janison R, “A DFT-based approach for efficient harmonic/inter-harmonic analysis under time-varying conditions," in Proc. Power and Energy Society General Meeting-Conversion and Delivery of Electrical Energy in the 21st Century, pp. 1-7, 2008.

[14] Dash, P. K, and A. M. Sharaf, "A Kalman filtering approach for estimation of power system harmonics," in Proc. The 3rd Int. Conf. Harmonics Power Syst., pp. 34-40, 1988.

[15] Al-Feilat, Eyad A, Abu, Ibrahim El-Amin, Maamar, and Bettayeb, "Power system harmonic estimation: a comparative study," Electric power systems research, Vol. 29, No. 2, pp. 91-97, Mar. 1994.

[16] Bettayeb, Maamar, and U. Qidwai, "Recursive estimation of power system harmonics," Electric power systems research, Vol. 47, No. 2, pp. 143-152, Oct. 1998.

[17] IEC 61000-4-7, Electromagnetic compatibility (EMC)Part 4-7: Testing and measurement technique- General guide on harmonics and interharmonics measurements and instrumentation, for power supply systems and equipment connected thereto, 2002.

[18] Chen, Kui Fu, and $\mathrm{Shu} \mathrm{Li}$ Mei, "Composite interpolated fast Fourier transform with the Hanning window," IEEE Trans. Instrum. Meas., Vol. 59, No. 6, pp. 1571-1579, 2010.

[19] Lin, Hsiung Cheng. "Accurate Harmonic / Interharmonic Estimation Using DFT-Based GroupHarmonics Energy Diffusion Algorithm," Canadian Journal of Electrical and Computer Engineering, Vol. 36, No. 4, pp. 158-171, 2013.

[20] H. Fujita, and H. Akagi, "A practical approach to harmonic compensation in power systems-series connection of passive and active filters," IEEE Trans. Ind. Appl., Vol. 27, No. 6, pp. 1020-1025, Nov. 1991.

[21] K. L. Lo, and C. Zhang, "Decomposed three-phase power flow solution using the sequence component frame," in Proc. Generation, Transmission and Distribution, IEE Proceedings C, pp. 181-188, 1993.

[22] J. O. Rawlings, S. G. Pantula, and D. A. Dickey,
Applied Regression Analysis, New York: SpringerVerlag, 1998.

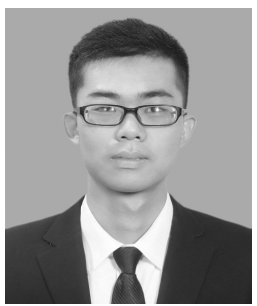

Ya Chen He received B.S degree in electrical engineering from Nanjing University of Science and Technology. And he is currently pursuing his M.S degree in South China University of Technology. His research interests are power harmonic estimation, protective relaying in power systems and power

quality.

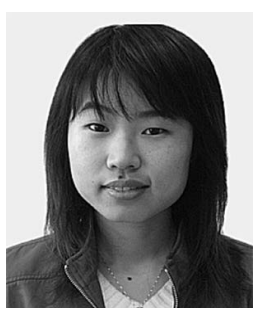

Tianyao Ji She received the $\mathrm{B}$. S degree in information engineering in 2003, the B.A. degree in English in 2003, and the M.S degree in signal and information processing in 2006 from Xi'an Jiaotong University, Xi'an, China. She received the Ph.D. degree in electrical engineering and electronics from the University of Liverpool, Liverpool, U.K., in 2009. From 2010 to 2011, she was a Research Associate with the University of Liverpool, Liverpool, U.K. Currently, she is an Associate Professor in the School of Electric Power Engineering, South China University of Technology, Guangzhou, China. Her research interests include mathematical morphology, signal and information processing, evolutionary computation, and their applications in power systems.

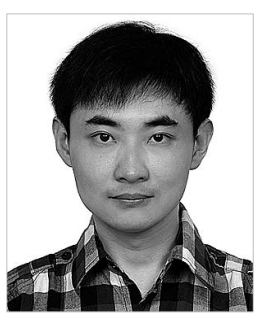

Mengshi Li He received the M.S degree in information and intelligence engineering from the University of Liverpool, Liverpool, U.K. and the Ph.D. degree in electrical engineering from the University of Liverpool, Liverpool, U.K., in 2005 and 2010, respectively. Currently, he is a Lecturer in the School of Electric Power Engineering, South China University of Technology, Guangzhou, China. His research interests include computational intelligence and their applications in power systems.

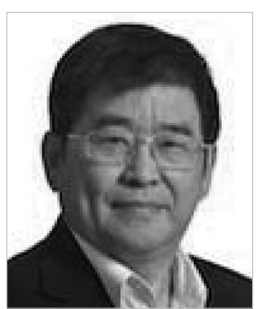

Qinghua Wu He received the Ph.D. degree in electrical engineering from The Queen's University of Belfast (QUB), Belfast, UK, in 1987. He was a Research Fellow and subsequently a Senior Research Fellow with QUB from 1987 to 1991, and as a Lecturer, and subsequently appointed Senior 
Lecturer in the Department of Mathematical Sciences, Loughborough University, Loughborough, UK, from 1991 to 1995. In 1995, he joined The Uvniversity of Liverpool, Liverpool, UK to become Chair of Electrical Engineering in the Department of Electrical Engineering and Electronics. Since then, he has been the Head of Intelligence Engineering and Automation Research Group, working in the areas of systems control, computational intelligence, as well as electric power and energy. $\mathrm{He}$ is also a Distinguished Professor at the South China University of Technology, Guangzhou, China. He has authored and coauthored more than 420 technical publications, including 190 journal papers, 20 book chapters, and 3 research monographs. His research interests include nonlinear adaptive control, mathematical morphology, evolutionary computation, machine learning, and power system control and operation.

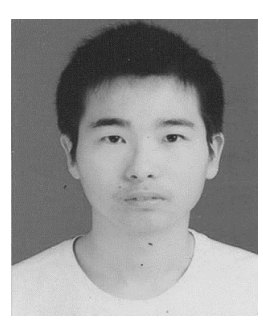

Xuejian Wang He received his B.S degrees in Electrical Engineering from North University of China in 2013. He is currently pursuing his M.S degree in South China University of Technology. His research interests include power quality analysis, power system signal processing. 